\title{
Optic Disk Boundary Detection
}

\author{
Simon Lee and Michael Brady
}

\author{
Robotics Research Group, \\ Department of Engineering Science, \\ University of Oxford
}

\begin{abstract}
Monitoring the shape changes of the optic disk is crucial for the early detection of glaucoma. This requires the optic disk to be segmented from the surrounding fundus. Previous methods required the boundary to be identified manually, for example, by specifying four control points on the boundary. This is not practical for routine clinical analysis, intra-observer and inter-observer variations are tend to be large. We develop an automatic boundary detection technique based on morphological filtering and an active contour model.
\end{abstract}

\section{Introduction}

In our last paper [1], we reported progress toward a system to aid ophthalmologists monitor the development of glaucoma, a complex of eye diseases that accounts for $12 \%$ of total registered blindness in the U.K. Structural changes in the optic disk is an important clinical feature, which involves the deepening and widening of the optic cup. The usual clinical practice to detect such changes is to measure the volume and depth of the optic cup, the vertical and horizontal cup/disk ratio and the neuroretinal rim area (non-cupping area of the optic disk). The surface of the optic disk can be reconstructed by integrating photometric stereo and binocular stereo [1]. The volume and depth of the optic cup are then computed directly from the reconstructed surface. The remaining problem is the determination of the optic disk boundary. We investigate an automatic method to identify the disk boundary, based on an active contour model.

Figure 1a) shows an image of the optic disk. The central circular structure is the optic disk. It can be seen that the optic disk is lighter in colour because of the lack of the pigmented epithelium layer. There are few blood vessels going into and out of the disk. The physical size of the optic disk is $1.8 \mathrm{~mm}$ in diameter on average and the boundary is roughly circular, but can be irregular in some disks.

Energy-minimising active contour models (snakes) have been used successfully in the past to detect irregular object boundaries by Kass et al. [2] and Amini et al. [3]. The images they worked on have relatively well defined boundaries, for example, a soda can against a uniform background. Unfortunately, fundus images are more complicated. The contrast between the optic disk and the fundus background is sometimes poor, even expert clinicians have difficulties in defining the exact position of the disk boundary in these regions. A more serious problem is the presence of the high contrast blood vessels. The blood vessels can distract the 
snake seriously when it tries to locate the disk boundary because it has larger image force (Section 3). Therefore the effect of the blood vessels should first be minimised. One possible solution is to remove the blood vessels; for example, using mathematical morphological closing operation. The snake could then be applied to the morphologically "closed" image where the boundary is more easily detectable.

\section{Mathematical Morphology}

Mathematical Morphological filter is described in [4]. The closing operation can be thought of as sliding a structuring element across the entire surface. The "closed" surface is defined as the lowest points reached by the structuring element. Therefore morphological closing effectively fits the surface locally. The fitted surface is defined by the structuring element, subject to the constraint that the resultant surface is equal to or above the original surface. The larger the structuring element, the better the interpolation across the blood vessels. The drawback is that computation time increases with the dimension of the structuring element and, more seriously, the edge of the boundary will be smoothed out if a large structuring element is used. Therefore an optimal structuring element is needed.

Ideally, the structuring element should be small enough to fit onto the disk boundary but large enough to interpolate across blood vessels. Since the blood vessels cross the disk boundary approximately perpendicularly, an elongated structuring element, such as an ellipse, will satisfy the above conflicting constraints. Different orientation of the structuring element have to be applied to different regions of the image. To simplify this, the intensity image in the Cartesian coordinate is transformed to polar $(r-\theta)$ coordinates. If the center of transformation lies inside the optic disk, the transformed boundary becomes an open curve in the polar plane. An elliptical structuring element, elongated in the $\theta$ direction, can be applied to the transformed image. The result of filtering is shown in Figure 1b). The radii of the ellipse used is 20 and 10 pixels, and the image size is $256 \times 256$. It can be seen that the blood vessels are removed and the disk boundary is continuous and profound.

\section{Implementation of the Snake}

In this section, we derive the energy function of the disk boundary snake. The energy is then minimised using the dynamic programming method. We incorporate three energies into our disk boundary snake - the internal energy, the spring energy and the image force.

1. Internal energy: Using the convention of Kass el al. [2], the snake is parameterised by $v(s)$, the internal energy can be described as

$$
E_{\text {smooth }}=\int \frac{d^{c} v}{d s^{c}} d s
$$

$c$ controls the continuity of the snake. We impose a very loose continuity constraint on the snake by only requiring it to be continuous, hence $c=1$. 
2. Spring energy: In order to provide some mechanism to pull the snake towards the disk boundary, springs are anchored between points on the disk boundary and the corresponding points on the snake. The spring energy is

$$
E_{\text {spring }}=k\left(v\left(s_{i}\right)-d_{i}\right)^{2}
$$

where $k$ is the spring constant and $d_{i}$ are the disk boundary locations where the springs are anchored. To identify the potential disk boundary points where the springs can be attached, a priori knowledge of the optic disk boundary are used. These include the expected size and position of the boundary.

3. Image energy: The image features that correspond to the disk boundary are high contrast edges in the image. Since the disk boundary is known to be roughly circular, only edges with appropriate direction are allowed to contribute to the total energy function. The image energy can be written as

$$
E_{\text {image }}=T(I)
$$

where

$$
T(I)=\left\{\begin{array}{cl}
-D\left(G_{\sigma} * I\right)^{2} & \text { if } D\left(G_{\sigma} * I\right)>0 \\
0 & \text { otherwise }
\end{array}\right.
$$

$D\left(G_{\sigma} * I\right)$ is a directional second derivative of the image intensity function $I$, and $G_{\sigma}$ is a Gaussian function. The direction of the second derivative is defined here as perpendicular to the tangent of the snake.

The total energy to be minimised is

$$
E_{t}=\alpha \int v_{s}^{2} d s+\beta \sum_{i}\left(v_{i}-d_{i}\right)^{2}+\gamma \int T(I) d s
$$

where $\alpha, \beta$ and $\gamma$ are the non-negative constant weights for $E_{\text {smooth }}, E_{\text {constraint }}$ and $E_{\text {image }}$ respectively. The energy function is discretised by the finite element method and is then minimised by the dynamic programming method [5] because this has the advantage of numerical stability and hard constraints can be imposed onto the energy minimisation process [3]. One hard constraint we imposed here is to restrict the movement of the control points on the snake in a direction towards the center of the image only. Since it is difficult to formulate an energy function that has a global minimum energy at the desired contour, the snake is made to be "myopic", that is, it will be trapped in local minimum. In order to achieve this, the initial position of the snake is set to the maximum expected size of the optic disk and it is then allowed to shrink. The search space is restricted to within 2 pixels for each control point; an optimised contour is then computed in each iteration and the disk boundary can be found within a finite number of iteration.

The result is shown in Figure 1c) with $\alpha=10, \beta=1 \gamma=50$. It can be seen that the boundary is accurately located. Various parameters values have been experimented with and it is found that the results are insensitive over wide ranges when the contrast of the disk boundary is large. On the other hand, when the contrast is poor, the snake is more sensitive to the choice of parameters since it is difficult to lock onto low contrast features. 


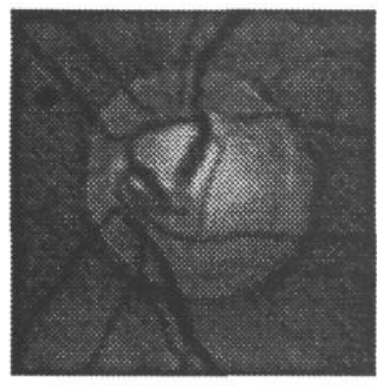

a

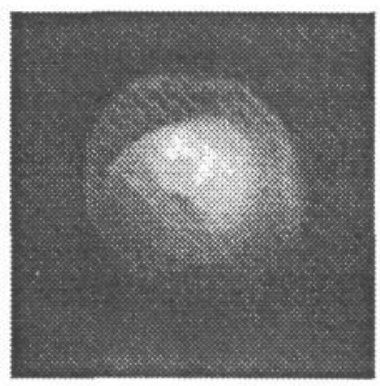

$\mathrm{b}$

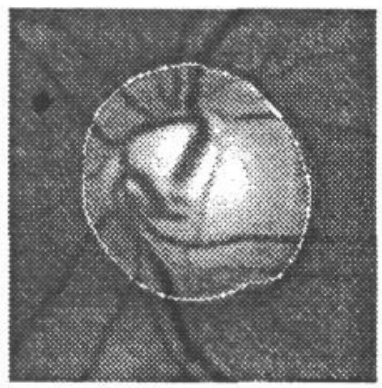

c

Figure 1: (a) shows an image of an optic disk. (b) shows the result of morphological closing operation. (c) shows the boundary (white curve) identified by the snake.

\section{Conclusions}

We described a method to identify the boundary of the optic disk. Since the blood vessels that cross the disk boundary pose a serious problem on the boundary detection process, we applied the morphological closing operation, using an application specific structuring element, to remove the blood vessels prior to the boundary detection process. The boundary can then be located accurately using a snake whose energy is minimised using the dynamic programming method.

\section{References}

[1] Lee S. and Brady J. M. Integrating stereo and photometric stereo to monitor the development of glaucoma. In Proceedings of the British Machine Vision Conference, pages 193-198, 1990.

[2] Kass M., Witkin A. P., and Terzopoulos D. Snake: Active contour models. In Proceedings of the First International Conference on Computer Vision, volume 1, pages 259-268, 1987.

[3] Amini A. A., Tehrani S., and Weymouth T. E. Using dynamic programming for minimization energy of active contour in the presence of hard constraints. In Proceedings of the Second International Conference on Computer Vision, pages 95-99, 1988.

[4] Serra J. Image Analysis and Mathematical Morphology. London: Academic, 1982.

[5] Bellman R. and Dreyfus S. Applied dynamic programming. Princeton University Press, Princeton, U.S., 1962. 